\title{
Robust Tracking Control of Robot Manipulators Using Only Joint Position Measurements
}

\author{
Ancai Zhang, ${ }^{1}$ Jinhua She, ${ }^{2}$ Xuzhi Lai, ${ }^{3}$ Min Wu, ${ }^{3}$ Jianlong Qiu, ${ }^{4}$ and Xiangyong Chen ${ }^{4}$ \\ ${ }^{1}$ School of Automobile Engineering, Linyi University, Linyi, Shandong 276005, China \\ ${ }^{2}$ School of Computer Science, Tokyo University of Technology, Hachioji, Tokyo 192-0982, Japan \\ ${ }^{3}$ School of Information Science and Engineering, Central South University, Changsha, Hunan 410083, China \\ ${ }^{4}$ School of Science, Linyi University, Linyi, Shandong 276005, China \\ Correspondence should be addressed to Jinhua She; she@stf.teu.ac.jp
}

Received 13 August 2013; Accepted 23 September 2013

Academic Editor: Bo-Chao Zheng

Copyright (C) 2013 Ancai Zhang et al. This is an open access article distributed under the Creative Commons Attribution License, which permits unrestricted use, distribution, and reproduction in any medium, provided the original work is properly cited.

\begin{abstract}
This paper concerns the tracking control of a robot manipulator with unknown uncertainties and disturbances. It presents a new control method that uses only joint position measurements to design a tracking controller. The controller has two parts. One is based on a feedback linearization technique; it makes the nominal model of a manipulator asymptotically track a desired trajectory. The other is based on the idea of equivalent input disturbance (EID); it compensates for uncertainties and disturbances. Together they enable a robot manipulator to precisely track the desired trajectory. The new control algorithm is applied to a two-link robot manipulator, and simulation results demonstrate the validity of this method.
\end{abstract}

\section{Introduction}

Robot manipulators are widely used in many fields. They are especially useful in areas where it is impractical or undesirable for a human to go, for example, undersea exploration, radioactive environments, and defusing explosive devices. Interest in the control of robot manipulators has been increasing over the past few years [1-3], and it is now a central issue in robotics.

If an exact dynamic model of a robot manipulator is known, the motion control problem is easy to solve by the computed-torque-control (CTC) method [4]. It uses nonlinear state feedback to cancel the nonlinear terms and a simple PD controller for motion control. Although this method is simple and effective, the requirement of an exact model limits its practicality because it is usually impossible to obtain an exact, or even reasonably accurate, dynamic model in practical applications. For example, an actual plant inevitably contains structured and unstructured uncertainties, and a robot manipulator may be influenced by unpredictable external disturbances when the operating environment changes. Since these uncertainties and disturbances may greatly affect control performance, it is necessary to consider their effects in the study of the motion control of robot manipulators.

A number of strategies have been developed to solve the problem of controlling the motion of a robot manipulator with uncertainties and disturbances. They include a Lyapunov-based method [5], a neural-network-based method [6, 7], an adaptive neural network $H_{\infty}$ strategy [8], an adaptive switching learning PD (ASL-PD) method [9], a parameterdependent nonlinear observer approach [10], and a variablestructure PID control method [11]. However, all of them require measurement of both the displacement and velocity of joints.

Generally speaking, joint displacement can be accurately measured with an encoder. However, velocity is typically measured with a tachometer, and the results usually contain noise, which can affect the control precision and performance of a closed-loop system. So, both practically and theoretically, it is meaningful to devise a motion control method for robot manipulators which relies only on the measurement of joint position. Various strategies have been developed to solve this challenging problem. One is a controller-observer combination strategy. It has a two-step design procedure: 
(1) construct an observer for a robot manipulator based on joint information and (2) use joint displacement and the state of the observer to design a state feedback controller. Many control methods based on this strategy have been reported [12-14]. The problem with it is that the stability of both the observer and the controller is of a local nature. Another strategy [15-17] involves using a linear or nonlinear compensator to obtain substitutes for the velocity variables. It enables the global tracking control of a robot manipulator, but the addition of external state variables complicates the design of the control system. A third is an adaptive tracking control strategy that includes an output feedback scheme (OFS) [18] and an iterative learning scheme (ILS) [19], but the OFSbased controller only locally ensures the asymptotic stability of the joint position error, and the ILS-based controller makes the system only track the same task iteratively.

This paper presents a new tracking control approach for a robot manipulator with unknown uncertainties and disturbances. Its advantage is that the design of the tracking controller relies only on the measurements of joint position, not velocity, and the tracking control is global. It is based on the concept of an equivalent input disturbance (EID), which was first presented in [20] to deal with disturbance rejection in a linear servo system. The EID approach has been validated through application to several mechatronic systems [20-22]. In this study, it was used to design a global robust tracking controller for a robot manipulator. The controller has two parts: one makes the nominal model of a manipulator asymptotically track a desired trajectory and the other compensates for uncertainties and disturbances. Together they enable a robot manipulator with unknown uncertainties and disturbances to precisely track the desired trajectory.

The rest of this paper is organized as follows. Section 2 describes the model and formulates the problem. Section 3 explains the design of an EID-based tracking controller. Section 4 discusses a numerical example for a two-link robot manipulator. Finally, Section 5 presents some concluding remarks.

\section{Model Description and Problem Formulation}

For a robot manipulator with $n$ serial links, we take $\ddot{q}, \dot{q}, q \in$ $\mathbb{R}^{n}$ to be the acceleration, velocity, and position vectors, respectively, of the joints. Choose the Lagrangian of the robot system to be

$$
L(q, \dot{q})=\frac{1}{2} \dot{q}^{T} M(q) \dot{q}-P(q)
$$

where $M(q) \in \mathbb{R}^{n \times n}$ is a positive-definite symmetric inertia matrix and $P(q)$ is the potential energy of the system. The equation of motion of the manipulator is obtained from the Euler-Lagrange equation:

$$
\frac{d}{d t}\left[\frac{\partial L(q, \dot{q})}{\partial \dot{q}}\right]-\frac{\partial L(q, \dot{q})}{\partial q}=\tau-\tau_{f}+\tau_{\mathrm{ext}},
$$

where $\tau \in \mathbb{R}^{n \times 1}$ is the vector of control torques, $\tau_{f} \in \mathbb{R}^{n \times 1}$ is the vector of friction torques, and $\tau_{\text {ext }} \in \mathbb{R}^{n \times 1}$ is the vector of the external disturbances imposed on the joints. We rewrite (2) in the general form

$$
M(q) \ddot{q}+H(q, \dot{q})+N(q)=\tau-\tau_{f}+\tau_{\text {ext }},
$$

where $H(q, \dot{q}) \in \mathbb{R}^{n \times 1}$ is the vector of Coriolis and centrifugal forces and $N(q) \in \mathbb{R}^{n \times 1}$ is the gravity vector.

Due to the unmodeled dynamics, measurement error, and changes in environment, it is difficult to obtain precise values for the masses and lengths of the links, the moments of inertia of the links, and other physical parameters. The measured values of these parameters are usually not very accurate. Thus, the values of the matrices $M(q), H(q, \dot{q})$, and $N(q)$ in (3) are

$$
\begin{gathered}
M(q)=M_{0}(q)+\Delta M(q), \\
H(q, \dot{q})=H_{0}(q, \dot{q})+\Delta H(q, \dot{q}), \\
N(q)=N_{0}(q)+\Delta N(q),
\end{gathered}
$$

where $M_{0}(q), H_{0}(q, \dot{q})$, and $N_{0}(q)$ are the nominal values of $M(q), H(q, \dot{q})$, and $N(q)$, respectively, and $\Delta M(q), \Delta H(q, \dot{q})$, and $\Delta N(q)$ are the corresponding additive uncertain terms. Consequently, (3) becomes

$$
M_{0}(q) \ddot{q}+H_{0}(q, \dot{q})+N_{0}(q)=\tau+d
$$

where

$$
d=\tau_{\text {ext }}-\Delta M(q) \ddot{q}-\Delta H(q, \dot{q})-\Delta N(q)-\tau_{f} .
$$

We assume that there is no prior information about $\Delta M(q), \Delta H(q, \dot{q}), \Delta N(q), \tau_{f}$, or $\tau_{\text {ext }}$. Thus, $d$ is an unknown disturbance of the nonlinear system (5).

Let $q_{r} \in \mathbb{R}^{n \times 1}$ be the desired trajectory of the manipulator, and let $e=q-q_{r}$ be the tracking error of the trajectory. If we take the control law, $\tau$, in (5) to be

$$
\begin{aligned}
\tau_{0}= & M_{0}(q)\left[\ddot{q}_{r}-K_{D}\left(\dot{q}-\dot{q}_{r}\right)-K_{P}\left(q-q_{r}\right)\right] \\
& +H_{0}(q, \dot{q})+N_{0}(q)
\end{aligned}
$$

where $K_{P}$ and $K_{D} \in \mathbb{R}^{n \times n}$ are two given positive-definite diagonal matrices, then (5) and (7) give

$$
\ddot{e}+K_{D} \dot{e}+K_{P} e-M_{0}^{-1}(q) d=0 .
$$

Assume that $\Delta M(q), \Delta H(q, \dot{q}), \Delta N(q), \tau_{f}$, and $\tau_{\text {ext }}$ are all zero, which means that there are no parameter perturbations and external disturbances acting on the manipulator. Then, combining (6) and (8) yields

$$
\ddot{e}+K_{D} \dot{e}+K_{P} e=0 .
$$

It is easy to obtain $\lim _{t \rightarrow \infty} e=0$ and $\lim _{t \rightarrow \infty} \dot{e}=0$ for (9). So, the state variables of the system, $\left[q^{T}, \dot{q}^{T}\right]$, asymptotically approach the desired trajectory, $\left[q_{r}^{T}, \dot{q}_{r}^{T}\right]$, for this case.

However, in practice it is difficult to acquire exact knowledge of a robot manipulator; uncertainties and disturbances can greatly reduce the tracking precision. So, we need to consider the tracking control problem for the perturbed robot system (5). This paper presents an EID-based tracking controller for (5) that relies only on measured joint position, $q$. 


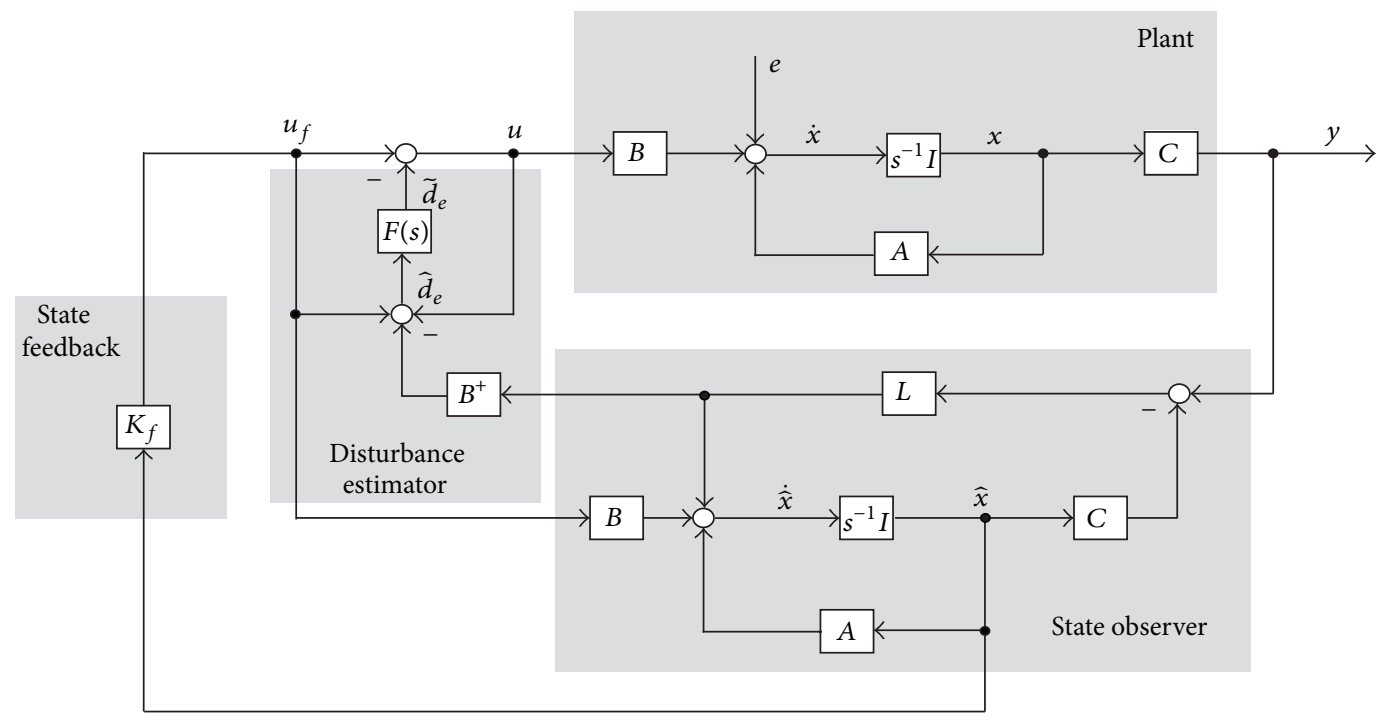

FIGURE 1: Configuration of an EID-based control system.

\section{Design of EID-Based Tracking Controller}

For the nonlinear system (5), we take

$$
\tau=\tau_{0}+M_{0}(q) \widetilde{B} u
$$

where $\tau_{0}$ is defined in (7); $\widetilde{B}=\left[b_{1}, b_{2}, \ldots, b_{n}\right]^{T} \in \mathbb{R}^{n \times 1}, b_{i}(i=$ $1,2, \ldots, n)$ are constants; and $u \in \mathbb{R}$ is a new input that is used to compensate for the disturbance, $d$. Combining (5) and (10) yields

$$
\ddot{e}=-K_{D} \dot{e}-K_{P} e+\widetilde{B} u+M_{0}^{-1}(q) d .
$$

Let $x=\left[e^{T}, \dot{e}^{T}\right]^{T} \in \mathbb{R}^{2 n \times 1}$; the state space form of (11) is

$$
\dot{x}=A x+B u+\varepsilon,
$$

where

$$
\begin{gathered}
A=\left[\begin{array}{cc}
0_{n \times n} & I_{n} \\
-K_{P} & -K_{D}
\end{array}\right], \quad B=\left[\begin{array}{c}
0_{n \times 1} \\
\widetilde{B}
\end{array}\right], \\
\varepsilon=\left[\begin{array}{c}
0_{n \times 1} \\
M_{0}^{-1}(q) d
\end{array}\right],
\end{gathered}
$$

where $I_{n}$ is the $n \times n$ identity matrix and $0_{i \times j}$ is an $i \times j$ zero matrix for positive integers $i$ and $j$.

Since only the measured $q$ is available, we take the output of (12) to be

$$
y=C x=e, \quad C=\left[I_{n}, 0_{n \times n}\right] .
$$

It is easy to verify that $(C, A)$ is observable. If we denote

$$
\Phi(s)=\left[K_{P}+\left(s I_{n}+K_{D}\right) s I_{n}\right]^{-1},
$$

then

$$
\begin{aligned}
C(s I-A)^{-1} B= & {\left[I_{n}, 0_{n \times n}\right] } \\
& \times\left[\begin{array}{cc}
-\Phi(s)\left(s I_{n}+K_{D}\right) & \Phi(s) \\
-I_{n}-s I_{n} \Phi(s)\left(s I_{n}+K_{D}\right) & s I_{n} \Phi(s)
\end{array}\right] B \\
& =\left[-\Phi(s)\left(s I_{n}+K_{D}\right), \Phi(s)\right] B \\
& =\left[0_{n \times 1},-\Phi(s) \widetilde{B}\right] .
\end{aligned}
$$

Equation (16) tells us that $(A, B, C)$ has no zeros on the imaginary axis because

$$
\Phi(s) \widetilde{B}=\left[\frac{b_{1}}{\phi_{1}(s)}, \frac{b_{2}}{\phi_{2}(s)}, \ldots, \frac{b_{n}}{\phi_{n}(s)}\right]^{T}
$$

where $\phi_{i}(s)=s^{2}+K_{d i} s+K_{p i}(i=1,2, \ldots, n)$, and $K_{p i}$ and $K_{d i}$ are the diagonal elements of the matrices $K_{P}$ and $K_{D}$, respectively.

In addition, we choose $b_{i}$ in $\widetilde{B}$ such that $(A, B)$ is controllable. Then, as shown in [20], there always exists an EID, $d_{e}$, on the control input channel. It produces the same effect on the output, $y$, as the disturbance, $\varepsilon$, does. The perturbed plant (12) and (14) can be considered to be an EID-based plant:

$$
\begin{gathered}
\dot{x}=A x+B\left[u+d_{e}\right], \\
y=C x .
\end{gathered}
$$

In the configuration of the EID-based control system (see Figure 1), $K_{f}$ is the feedback gain; $L$ is the observer gain; $\widehat{d}_{e}$ is an estimate of the EID, $d_{e}$; and $F(s)$ is a low-pass filter that limits the angular frequency band of the disturbance estimate. Their design is discussed below. 
3.1. Estimation of Equivalent Input Disturbance. First, we construct a full-order Luenberger observer for (18):

$$
\dot{\hat{x}}=A \widehat{x}+B u_{f}+L(y-C \widehat{x}),
$$

where $u_{f}=u+d_{e}$. Letting

$$
\Delta x=\widehat{x}-x
$$

and substituting that into (18) yield

$$
\dot{\hat{x}}=A \widehat{x}+B\left[u+\widehat{d}_{e}\right]
$$

where

$$
\begin{gathered}
\widehat{d}_{e}=d_{e}+\Delta d, \\
B \Delta d=\Delta \dot{x}-A \Delta x .
\end{gathered}
$$

Note that (21) has the same form as (18). We take $\widehat{d}_{e}$ to be an estimate of the actual EID, $d_{e}$. From (18), (21), and (22), it is clear that the difference between the state of the plant and that of the observer is equivalent to the difference between $d_{e}$ and $\widehat{d}_{e}$. Combining (19) and (21) yields

$$
B\left(u+\widehat{d}_{e}-u_{f}\right)=L(y-C \widehat{x}) .
$$

We solve (23) for $\widehat{d}_{e}$ and obtain a least-squares solution:

$$
\widehat{d}_{e}=B^{+} L(y-C \hat{x})+u_{f}-u
$$

where

$$
B^{+}=\left[B^{T} B\right]^{-1} B^{T}
$$

To ensure the estimation accuracy, the low-pass filter

$$
F(s)=\frac{1}{T s+1}
$$

is used to select the angular frequency band for disturbance estimation, where $T$ is the time constant. The filtered disturbance estimate, $\widetilde{d}_{e}$, is given by

$$
\widetilde{D}_{e}(s)=F(s) \widehat{D}_{e}(s),
$$

where $\widetilde{D}_{e}(s)$ and $\widehat{D}_{e}(s)$ are the Laplace transforms of $\widetilde{d}_{e}$ and $\widehat{d}_{e}$, respectively.

We take control law $u$ to be

$$
\begin{gathered}
u=u_{f}-\widetilde{d}_{e}, \\
u_{f}=K_{f} \widehat{x}
\end{gathered}
$$

where $K_{f}$ is a feedback gain that makes $A+B K_{f}$ stable. Assume that the observer (19) is stable. Then, control law (29) makes the output of plant (18) asymptotically converge to zero. According to the definition of EID, control law (28) asymptotically stabilizes the output of (12), which is $e$, at the origin. Thus, the tracking control objective of system (12) is achieved.
Remark 1. From (7) and (10), the velocity, $\dot{q}$, is needed to calculate the control input, $\tau$. Since we assume that the measured value of $\dot{q}$ is unavailable, we use information of the observer (19) to obtain a substitute for $\dot{q}$ in (7):

$$
\dot{q}=\widehat{x}_{2}+\dot{q}_{r}
$$

where $\widehat{x}_{2} \in \mathbb{R}^{n}, \widehat{x}=\left[\widehat{x}_{1}^{T}, \widehat{x}_{2}^{T}\right]^{T}$ is the state vector of the observer (19), and $\widehat{x}_{1} \in \mathbb{R}^{n}$.

3.2. Design of State Observer. Since the separation theorem holds for an EID-based control system [20], we can separately design the feedback gain $K_{f}$ and the observer gain $L$. Since $(A, B)$ is controllable, it is easy to design a $K_{f}$ by any appropriate method (pole placement, optimal control, etc.). So, here we focus on the design of $L$.

The design of $L$ should first ensure the stability of the state observer (19). We take

$$
\begin{gathered}
L=K_{\rho}^{T}, \\
K_{\rho}=R_{L}^{-1} C S, \\
A S+S A^{T}-S C^{T} R_{L}^{-1} C S+\rho Q_{L}=0,
\end{gathered}
$$

where $Q_{L}$ and $R_{L}$ are two given positive-definite matrices and $\rho$ is a positive scalar. Since $(C, A)$ is observable, $\left(C^{T}, A^{T}\right)$ is controllable. Thus, the observer gain, $L$, designed in (31) makes $A-L C$ stable, which means that the state observer (19) is stable.

On the other hand, we tackle the stability issue by first letting $d=0$. From (19), (21), and (22), we have

$$
\Delta \dot{x}=(A-L C) \Delta x+B \tilde{d}_{e} .
$$

Combining (24) and (10) yields

$$
\widehat{d}_{e}=-B^{+} L C \Delta x+\tilde{d}_{e} .
$$

From (32) and (24), we obtain the transfer function from $\widetilde{d}_{e}$ to $\widehat{d}_{e}$ :

$$
\begin{aligned}
G(s) & =1-B^{+} L C[s I-(A-L C)]^{-1} B \\
& =B^{+}(s I-A)[s I-(A-L C)]^{-1} B .
\end{aligned}
$$

Note that the transfer function from $\widehat{d}_{e}$ to $\widetilde{d}_{e}$ is $F(s)$ (Figure 2). The small-gain theorem [23] tells us that the condition

$$
\|G F\|_{\infty}<1
$$

must be satisfied to guarantee the stability of the control system, where $\|G F\|_{\infty}:=\sup _{0 \leq \omega \leq \infty} \sigma_{\max }[G(j \omega) F(j \omega)]$ and $\sigma_{\text {max }}(\cdot)$ is the maximum singular-value function.

Since the number of inputs of the plant $\left(A^{T}, C^{T}, B^{T}\right)$ is not less than the number of outputs, and since $A^{T}-C^{T} K_{\rho}$ is stable, according to [24, Theorems 1 and 3], we have

$$
\lim _{\rho \rightarrow \infty}\left\{\left[s I-\left(A-K_{\rho}^{T} C\right)\right]^{-1} B\right\}^{T}=0 .
$$

Note that $\left[s I-\left(A-K_{\rho}^{T} C\right)\right]^{-1} B$ is part of $G(s)$. So, for a given $F(s)$ in (26), the observer gain, $L$, designed in (31) makes the condition (35) true provided that $\rho$ is large enough. 


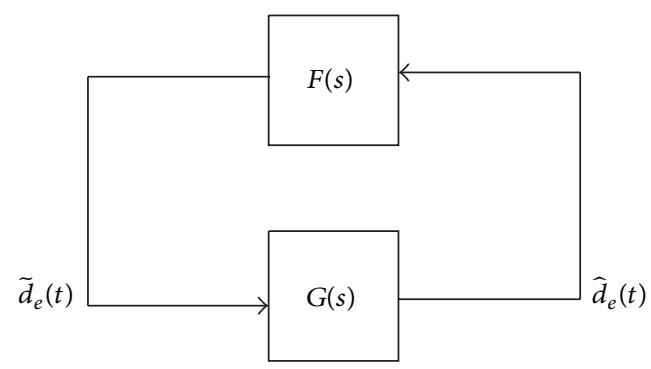

FIgURE 2: Stability condition (35).

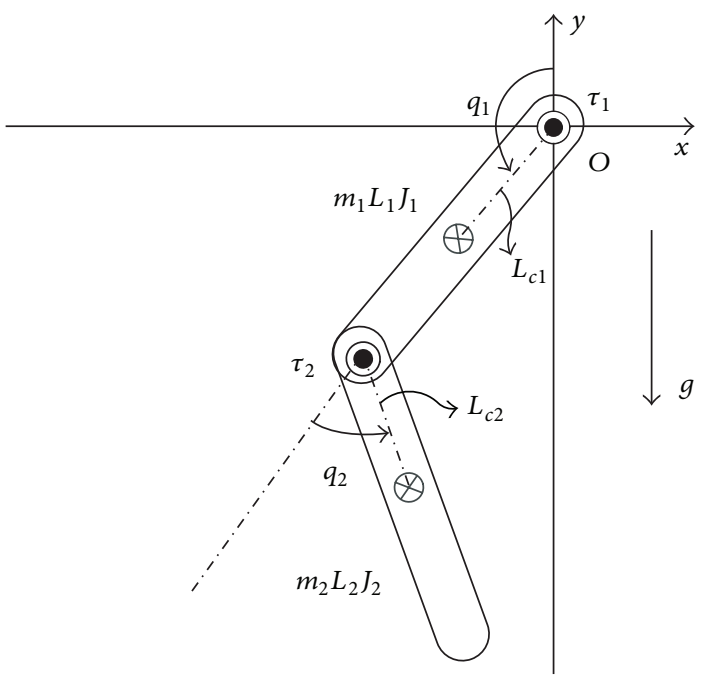

Actuator

$\otimes$ Center of mass

FIgURE 3: Two-link robot manipulator.

\section{Numerical Example}

We applied the EID-based tracking control strategy to a twolink rigid robot manipulator (Figure 3) to demonstrate its validity.

The simulations employed the parameters (Table 1) of a PUMA 560 manipulator [7]. The matrices for the dynamics of the nominal model are

$$
\begin{gathered}
M_{0}(q)=\left[\begin{array}{cc}
a_{1}+a_{2}+2 a_{3} \cos q_{2} & a_{2}+a_{3} \cos q_{2} \\
a_{2}+a_{3} \cos q_{2} & a_{2}
\end{array}\right], \\
H_{0}(q, \dot{q})=\left[\begin{array}{c}
-a_{3}\left(2 \dot{q}_{1}+\dot{q}_{2}\right) \dot{q}_{2} \sin q_{2} \\
a_{3} \dot{q}_{1}^{2} \sin q_{2}
\end{array}\right], \\
N_{0}(q)=\left[\begin{array}{c}
-b_{1} \sin q_{1}-b_{2} \sin \left(q_{1}+q_{2}\right) \\
-b_{2} \sin \left(q_{1}+q_{2}\right)
\end{array}\right],
\end{gathered}
$$

TABLE 1: Parameters of the two-link manipulator for simulations.

\begin{tabular}{lcccc}
\hline Link $i$ & $m_{i}(\mathrm{~kg})$ & $L_{i}(\mathrm{~m})$ & $L_{c i}(\mathrm{~m})$ & $J_{i}\left(\mathrm{~kg} \cdot \mathrm{m}^{2}\right)$ \\
\hline$i=1$ & 31.20 & 1.05 & 0.43 & 0.41 \\
$i=2$ & 22.53 & 0.78 & 0.32 & 0.35 \\
\hline
\end{tabular}

where

$$
\begin{gathered}
a_{1}:=m_{1} L_{c 1}^{2}+m_{2} L_{1}^{2}+J_{1}, \\
a_{2}:=m_{2} L_{c 2}^{2}+J_{2}, \\
a_{3}:=m_{2} L_{1} L_{c 2}, \\
b_{1}:=\left(m_{1} L_{c 1}+m_{2} L_{1}\right) g \\
b_{2}:=m_{2} L_{c 2} g,
\end{gathered}
$$

$q_{1}$ is the angle of the first link relative to the vertical, $q_{2}$ is the angle of the second link relative to the first link, $m_{i}$ is the mass of the $i$ th link ( $i=1,2), L_{i}$ is the length of the $i$ th link $(i=1,2), L_{c i}$ is the distance from the $i$ th joint to the center of mass (COM) of the $i$ th link $(i=1,2), J_{i}$ is the moment of inertia around the COM of the ith link $(i=1,2)$, $\tau_{i}$ is the torque applied to the $i$ th joint $(i=1,2)$, and $g$ is the gravitational constant $\left(9.80665 \mathrm{~m} / \mathrm{s}^{2}\right)$.

Let the desired trajectory $q_{r}=\left[q_{1 r}, q_{2 r}\right]^{T}$ be

$$
\begin{gathered}
q_{1 r}=0.5 \cos t+0.2 \sin 3 t, \\
q_{2 r}=-0.2 \sin 2 t-0.5 \cos t .
\end{gathered}
$$

First, when $d=0$, we choose

$$
K_{P}=\left[\begin{array}{ll}
1 & 0 \\
0 & 1
\end{array}\right], \quad K_{D}=\left[\begin{array}{ll}
2 & 0 \\
0 & 1
\end{array}\right]
$$

for (7) and (9). Figure 4 shows the tracking control results for the initial condition

$$
\left[q_{1}, q_{2}, \dot{q}_{1}, \dot{q}_{2}\right]^{T}=[\pi, 0,0,0]^{T} .
$$

Notice that the actual trajectories converge to the desired trajectories in less than 10 seconds.

Next, we consider the uncertainties in the physical parameters by letting $m_{1}$ and $L_{c 2}$ be $5 \%$ larger than their nominal values, letting $m_{2}$ and $L_{c 1}$ be $5 \%$ smaller than their nominal values, and letting $J_{1}$ and $J_{2}$ be $10 \%$ larger than their nominal values. We also added two types of torques to the joints of the manipulator: (a) a viscous friction torque, $\tau_{f}=\left[\tau_{1 f}, \tau_{2 f}\right]^{T}:$

$$
\tau_{1 f}=0.5 \dot{q}_{1}, \quad \tau_{2 f}=0.5 \dot{q}_{2},
$$

and (b) an external disturbance torque, $\tau_{\text {ext }}=\left[\tau_{1 \text { ext }}, \tau_{2 \text { ext }}\right]^{T}$ (Figure 5):

$$
\begin{gathered}
\tau_{1 \mathrm{ext}}=0.5(\sin 4 \pi t+\cos 2 \pi t), \\
\tau_{\text {2ext }}=0.5(\sin \pi t+\cos 0.5 \pi t) .
\end{gathered}
$$




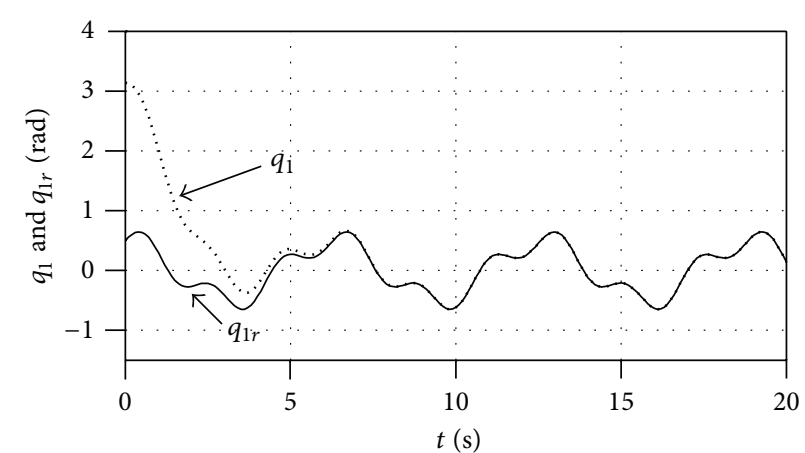

(a)

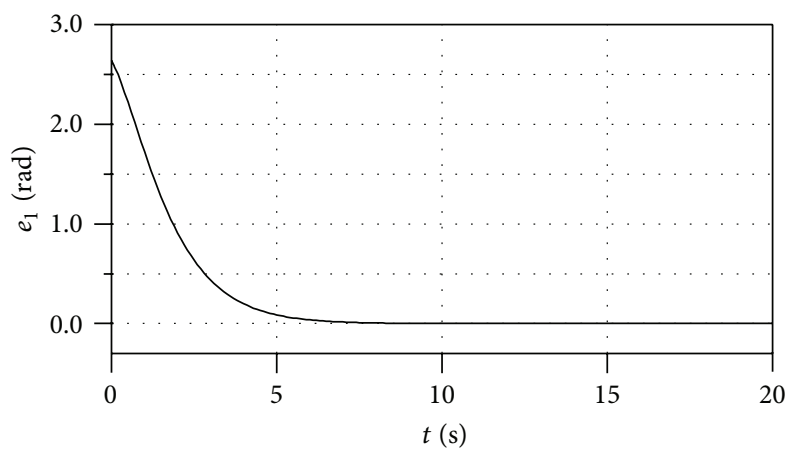

(c)

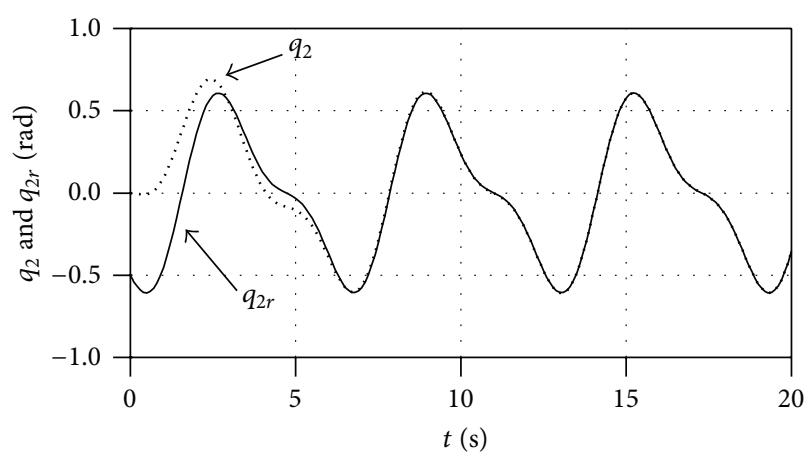

(b)

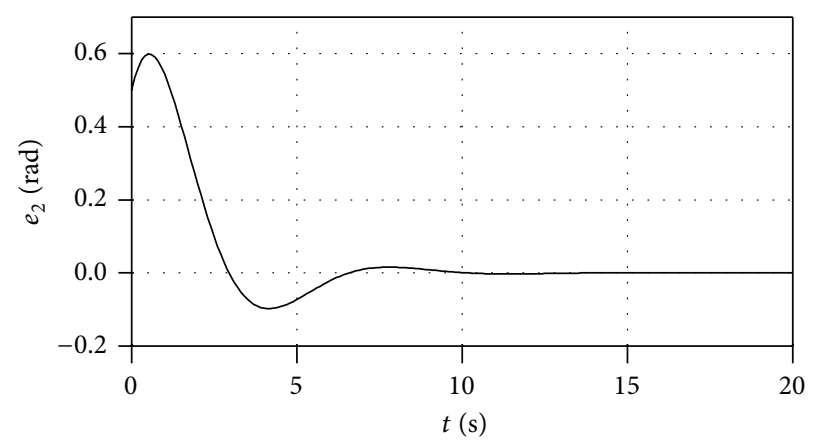

(d)

FIGURE 4: Tracking control results without uncertainties or disturbances.

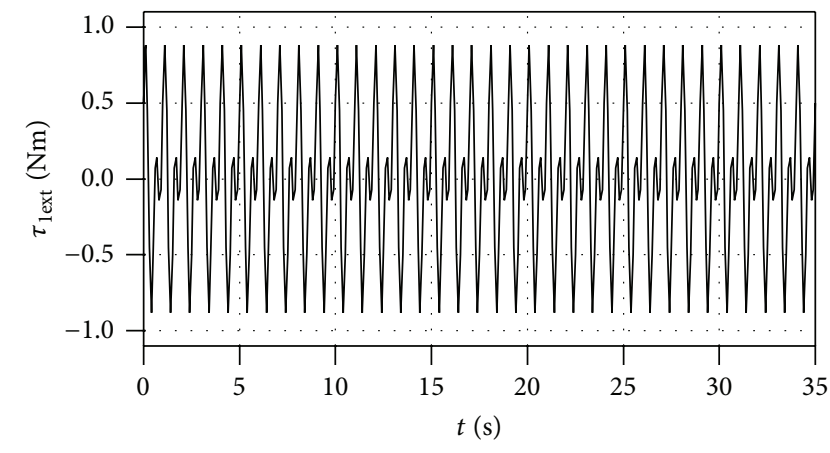

(a)

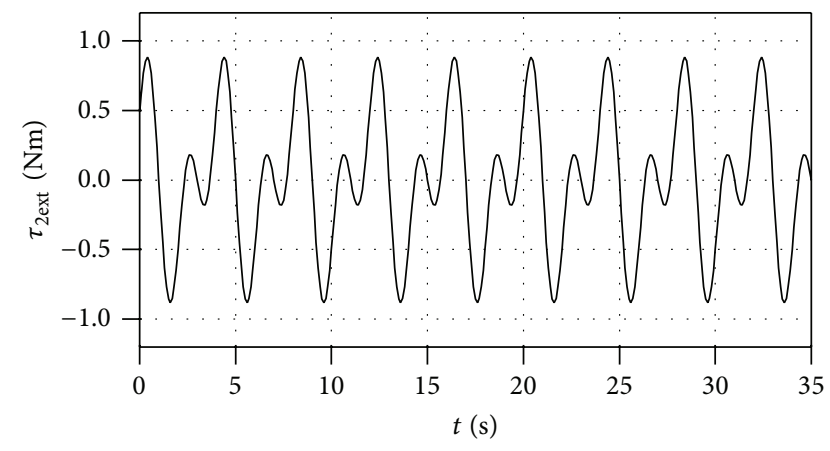

(b)

FIgURE 5: Disturbance to torque of joints added to the two-link manipulator.

The simulation results (Figure 6) for (5), (7), (40), and (41) show that the tracking performance was much worse, and a large steady-state tracking error appeared. That means that control law (7) by itself does not force the robot manipulator to track the desired trajectory when uncertainties and disturbances are present.

Finally, we applied the EID-based control strategy. We chose $\widetilde{B}=[10,10]$. It is easy to verify that $(A, B)$ is controllable. Letting all of the poles of $A+B K_{f}$ be -2.5 yields the feedback gain

$$
K_{f}=[-2.3625,-1.4437,-1.8563,1.1563] .
$$

The design parameters for (26) and (31) were chosen to be

$$
T=0.1, \quad Q_{L}=10 I_{4}, \quad R_{L}=50 I_{2}, \quad \rho=10^{6} .
$$

That gave

$$
\begin{gathered}
L=\left[\begin{array}{cccc}
447.4463 & 0 & 104.1078 & 0 \\
0 & 447.6245 & 0 & 183.8319
\end{array}\right]^{T}, \\
\|G F\|_{\infty}=0.9186<1 .
\end{gathered}
$$

The simulation results (Figure 7) for (5), (10), (28), (40), (41), and (45) show that the tracking performance was much 


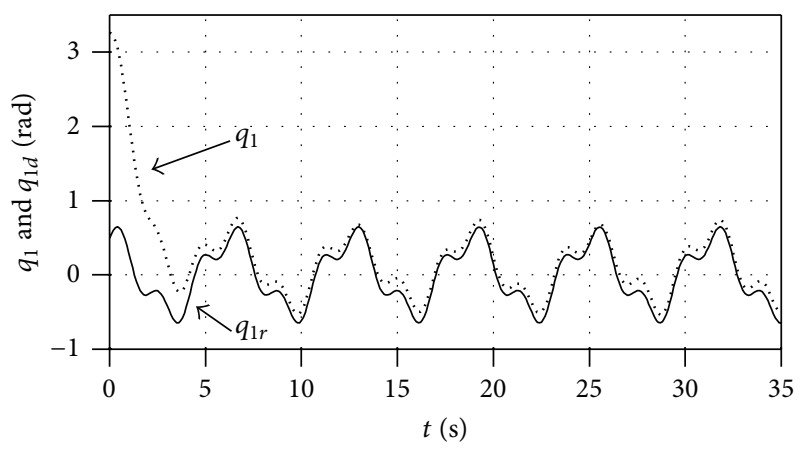

(a)

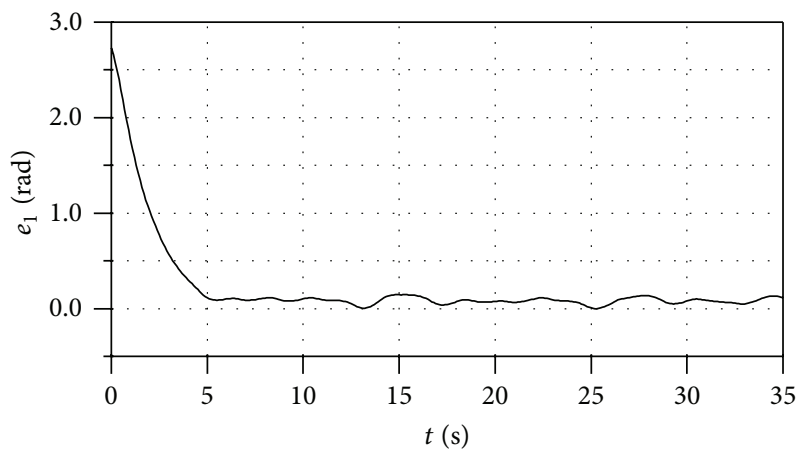

(c)

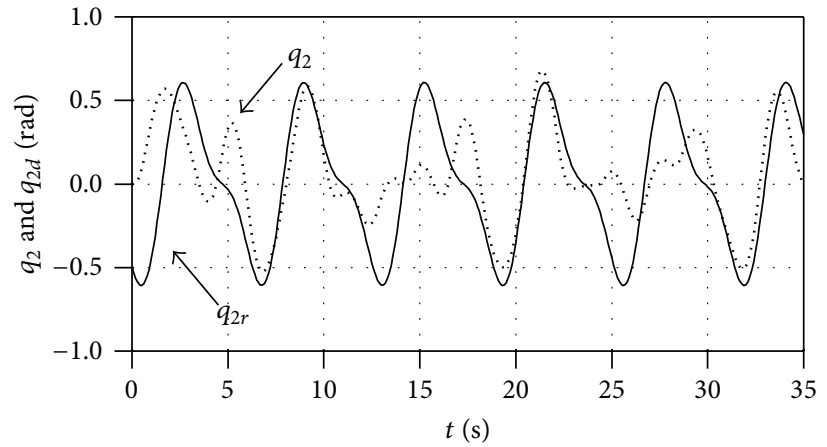

(b)

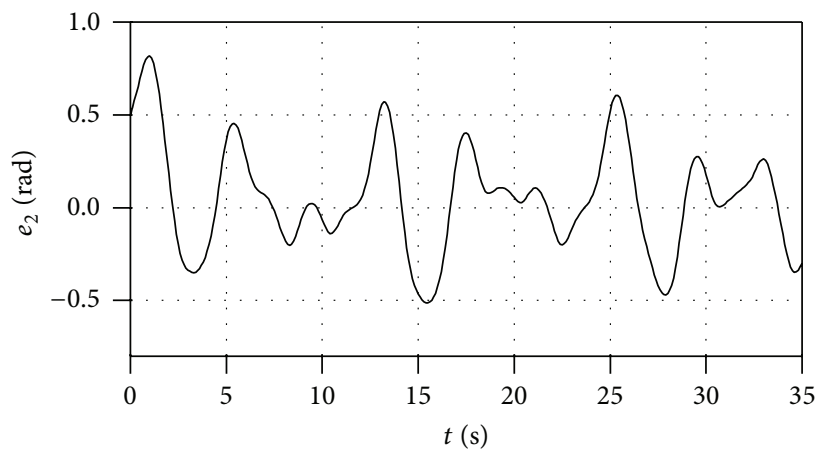

(d)

FIGURE 6: Simulation results for control law (7) when uncertainties and disturbances are present.

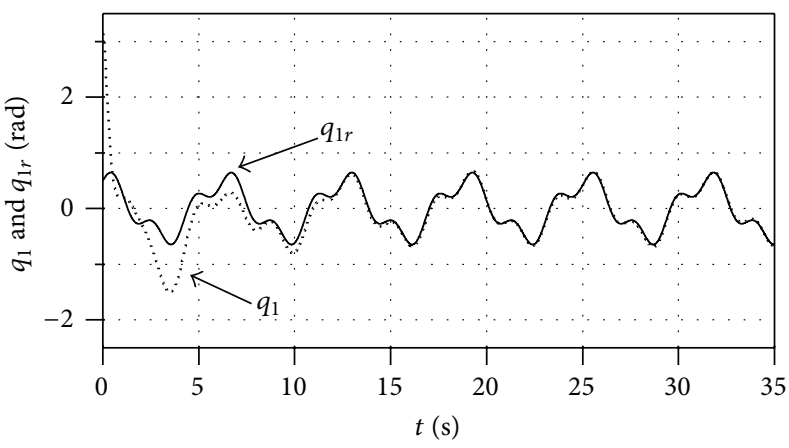

(a)

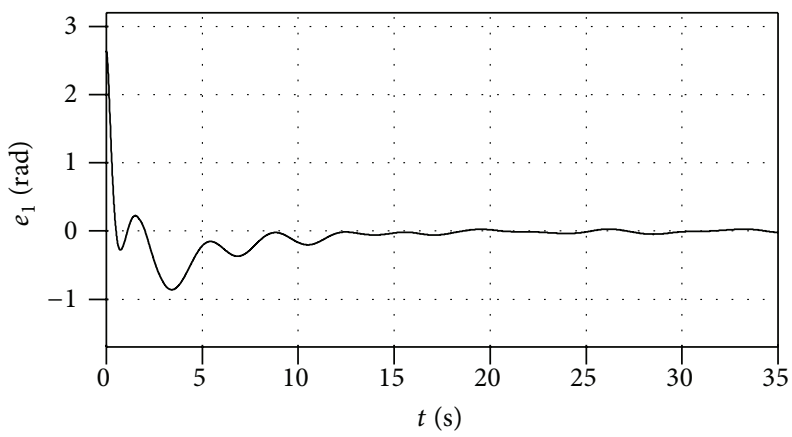

(c)

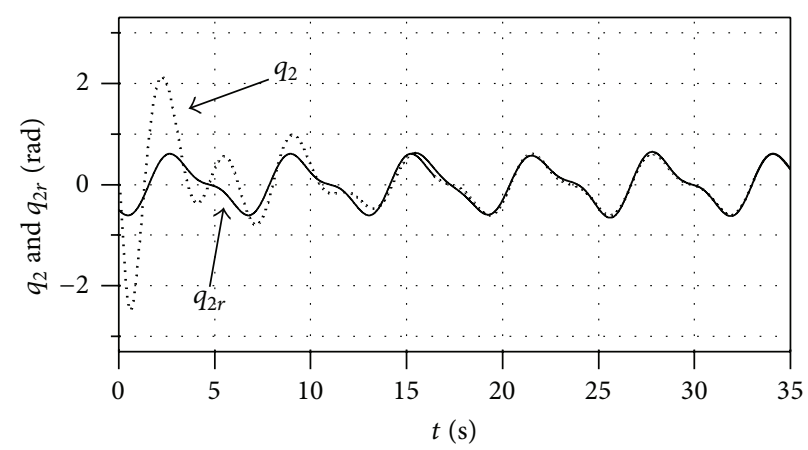

(b)

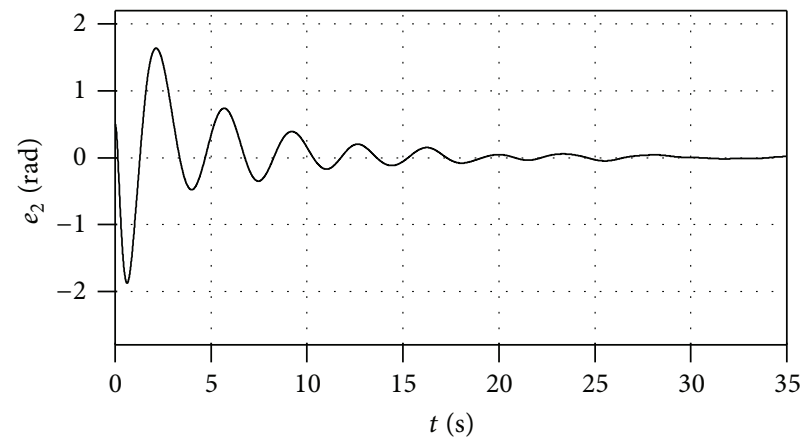

(d)

FIGURE 7: Simulation results for EID-based control when uncertainties and disturbances are present. 


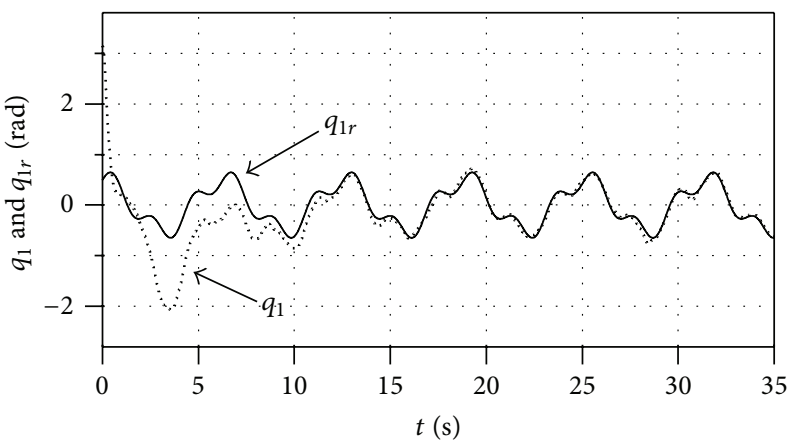

(a)

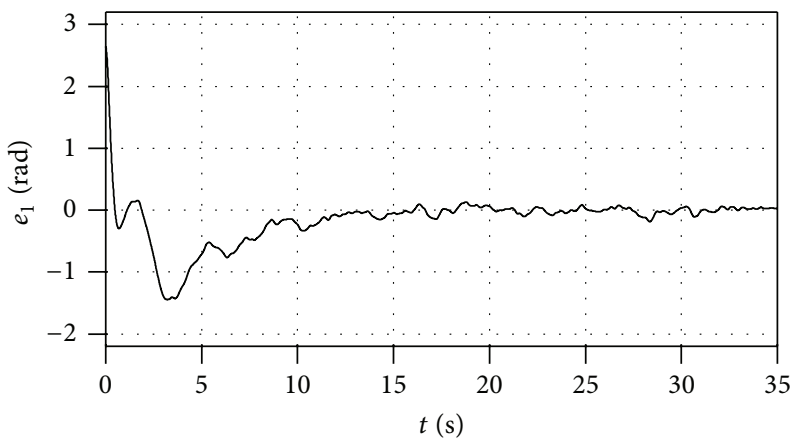

(c)

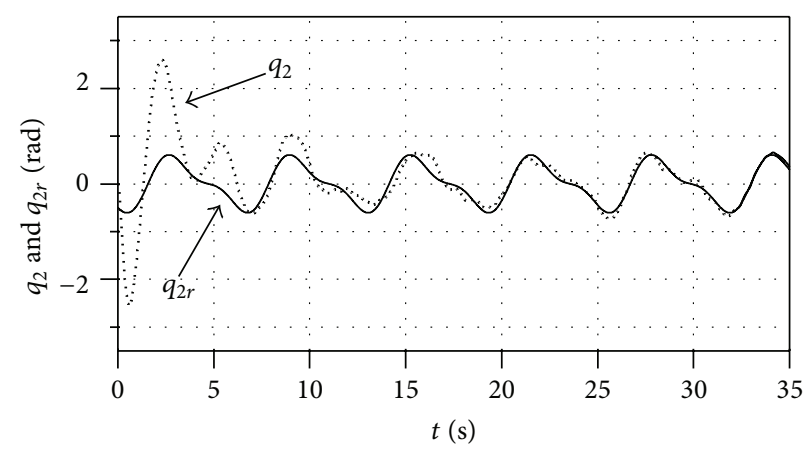

(b)

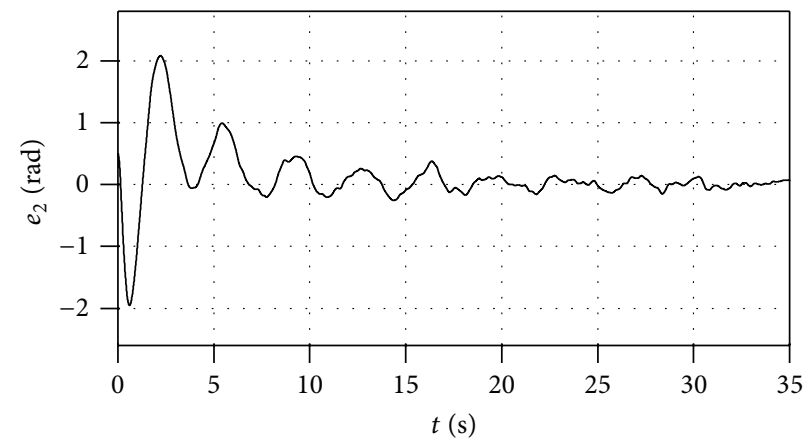

(d)

FIGURE 8: Simulation results for EID-based control when uncertainties, disturbances, and measurement white noise are present.

better than that in Figure 6. The EID-based controller (28) almost completely rejects the effect of the uncertainties and disturbances. So, the perturbed manipulator system (3) precisely tracks the desired trajectory. Since measurement noise is very common in actual control engineering applications, we also added white noise (peak value: \pm 0.2 ) to the measured $q$. The simulation results (Figure 8) show that the robot manipulator precisely tracks the desired trajectory even in this case.

\section{Conclusion}

This paper presents an EID-based control strategy that solves the tracking control problem for a robot manipulator with unknown uncertainties and disturbances. It uses only joint position measurements in the design of the tracking controller. The controller has two parts: one part uses an exact linearization technique to guarantee the asymptotical stability of the nominal model and the other is based on the idea of EID, which compensates for the effects of parameter uncertainties and exogenous disturbances. The combination makes a robot manipulator precisely track the desired trajectory. Simulation results show the validity of this control strategy.

\section{Acknowledgments}

This work was supported in part by the National Science Foundation of China under Grants nos. 61304023, 61273012 and 61074112, by the Project of Shandong Province Higher
Educational Science and Technology Program under Grant nos. J12LI58 and J13LI11, and by the Applied Mathematics Enhancement Program of Linyi University. The authors declare that there is no conflict of interests regarding the publication of this paper.

\section{References}

[1] B. Jayawardhana and G. Weiss, "Tracking and disturbance rejection for fully actuated mechanical systems," Automatica, vol. 44, no. 11, pp. 2863-2868, 2008.

[2] C. Canudas-de-Wit and R. Kelly, "Passivity analysis of a motion control for robot manipulators with dynamic friction," Asian Journal of Control, vol. 9, no. 1, pp. 30-36, 2007.

[3] M. Homayounzade and M. Keshmiri, "On the robust tracking control of kinematically constrained robot manipulators," in Proceedings of the IEEE International Conference on Mechatronics (ICM '11), pp. 248-253, Istanbul, Turkey, April 2011.

[4] R. H. Middleton and G. C. Goodwin, "Adaptive computed torque control for rigid link manipulations," Systems and Control Letters, vol. 10, no. 1, pp. 9-16, 1988.

[5] M. W. Spong, "On the robust control of robot manipulators," IEEE Transactions on Automatic Control, vol. 37, no. 11, pp. 17821786, 1992.

[6] R.-J. Wai, “Tracking control based on neural network strategy for robot manipulator," Neurocomputing, vol. 51, pp. 425-445, 2003.

[7] Y. Zuo, Y. Wang, X. Liu et al., "Neural network robust $H_{\infty}$ tracking control strategy for robot manipulators," Applied Mathematical Modelling, vol. 34, no. 7, pp. 1823-1838, 2010. 
[8] Y.-C. Chang and B.-S. Chen, "A nonlinear adaptive $H_{\infty}$ tracking control design in robotic systems via neural networks," IEEE Transactions on Control Systems Technology, vol. 5, no. 1, pp. 1329, 1997.

[9] P. R. Ouyang, W. J. Zhang, and M. M. Gupta, "An adaptive switching learning control method for trajectory tracking of robot manipulators," Mechatronics, vol. 16, no. 1, pp. 51-61, 2006.

[10] X. Wang, Y. Yao, and F. He, "Control design for harmonic disturbance rejection for robot manipulators with bounded inputs," in Proceedings of the Chinese Control and Decision Conference (CCDC '09), pp. 2323-2328, Guilin, China, June 2009.

[11] E. M. Jafarov, M. N. A. Parlakçi, and Y. Istefanopulos, "A new variable structure PID-controller design for robot manipulators," IEEE Transactions on Control Systems Technology, vol. 13, no. 1, pp. 122-130, 2005.

[12] S. Nicosia and P. Tomei, "Robot control by using only joint position measurements," IEEE Transactions on Automatic Control, vol. 35, no. 9, pp. 1058-1061, 1990.

[13] C. C. de Wit, N. Fixot, and K. J. Astrom, "Trajectory tracking in robot manipulators via nonlinear estimated state feedback," IEEE Transactions on Robotics and Automation, vol. 8, no. 1, pp. 138-144, 1992.

[14] M. Homayounzade, M. Keshmiri, and M. Danesh, "An observer-based state feedback controller design for robot manipulators considering actuators' dynamic," in Proceedings of the 15th International Conference on Methods and Models in Automation and Robotics (MMAR '10), pp. 240-248, Miedzyzdroje, Poland, August 2010.

[15] J. Alvarez-Ramirez, R. Kelly, and I. Cervantes, "Stable output feedback position control with integral action for robot manipulators," Asian Journal of Control, vol. 5, no. 2, pp. 230-241, 2003.

[16] H. Berghuis and H. Nijmeijer, "Robust control of robots via linear estimated state feedback," IEEE Transactions on Automatic Control, vol. 39, no. 10, pp. 2159-2162, 1994.

[17] A. Zavala-Río, E. Aguiñaga-Ruiz, and V. Santibáñez, “Global trajectory tracking through output feedback for robot manipulators with bounded inputs," Asian Journal of Control, vol. 13, no. 3, pp. 430-438, 2011

[18] S. Purwar, I. N. Kar, and A. N. Jha, "Adaptive output feedback tracking control of robot manipulators using position measurements only," Expert Systems with Applications, vol. 34, no. 4, pp. 2789-2798, 2008.

[19] S. Islam and P. X. Liu, "Adaptive iterative learning control for robot manipulators without using velocity signals," in Proceedings of the IEEE/ASME International Conference on Advanced Intelligent Mechatronics (AIM '10), pp. 1293-1298, Montreal, Canada, July 2010.

[20] J.-H. She, M. Fang, Y. Ohyama, H. Hashimoto, and M. Wu, "Improving disturbance-rejection performance based on an equivalent-input-disturbance approach," IEEE Transactions on Industrial Electronics, vol. 55, no. 1, pp. 380-389, 2008.

[21] J.-H. She, X. Xin, and Y. Ohyama, "Estimation of equivalent input disturbance improves vehicular steering control," IEEE Transactions on Vehicular Technology, vol. 56, no. 6, pp. 37223731, 2007.

[22] J.-H. She, X. Xin, and Y. Pan, "Equivalent-input-disturbance approachanalysis and application to disturbance rejection in dual-stage feed drive control system," IEEE/ASME Transactions on Mechatronics, vol. 16, no. 2, pp. 330-340, 2011.

[23] K. Zhou, J. Doyle, and K. Glover, Robust and Optical Control, Prentice Hall, Englewood Cliffs, NJ, USA, 1996.
[24] H. Kimura, "A new approach to the perfect regulation and the bounded peaking in linear multivariable control systems," IEEE Transactions on Automatic Control, vol. 26, no. 1, pp. 253-270, 1981. 


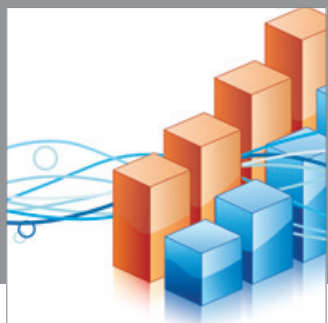

Advances in

Operations Research

mansans

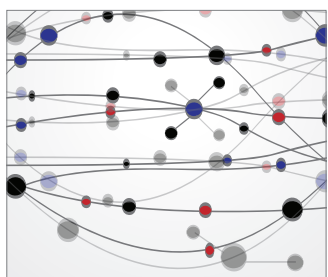

The Scientific World Journal
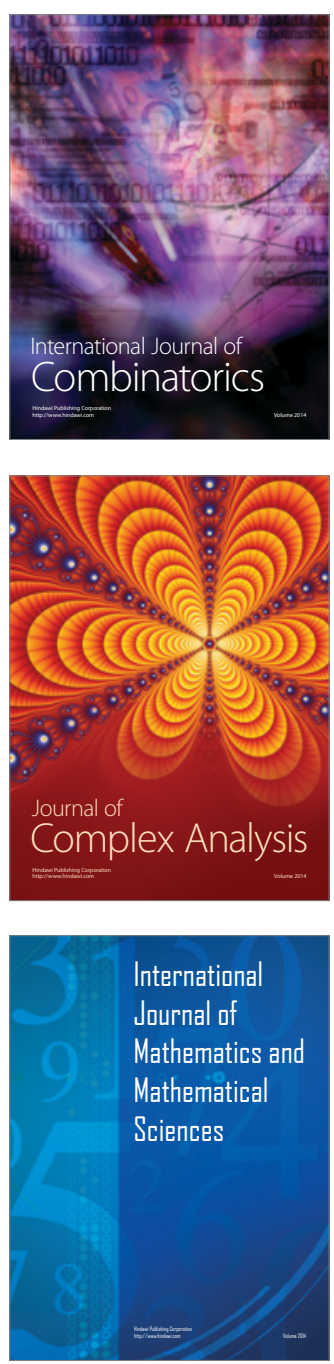
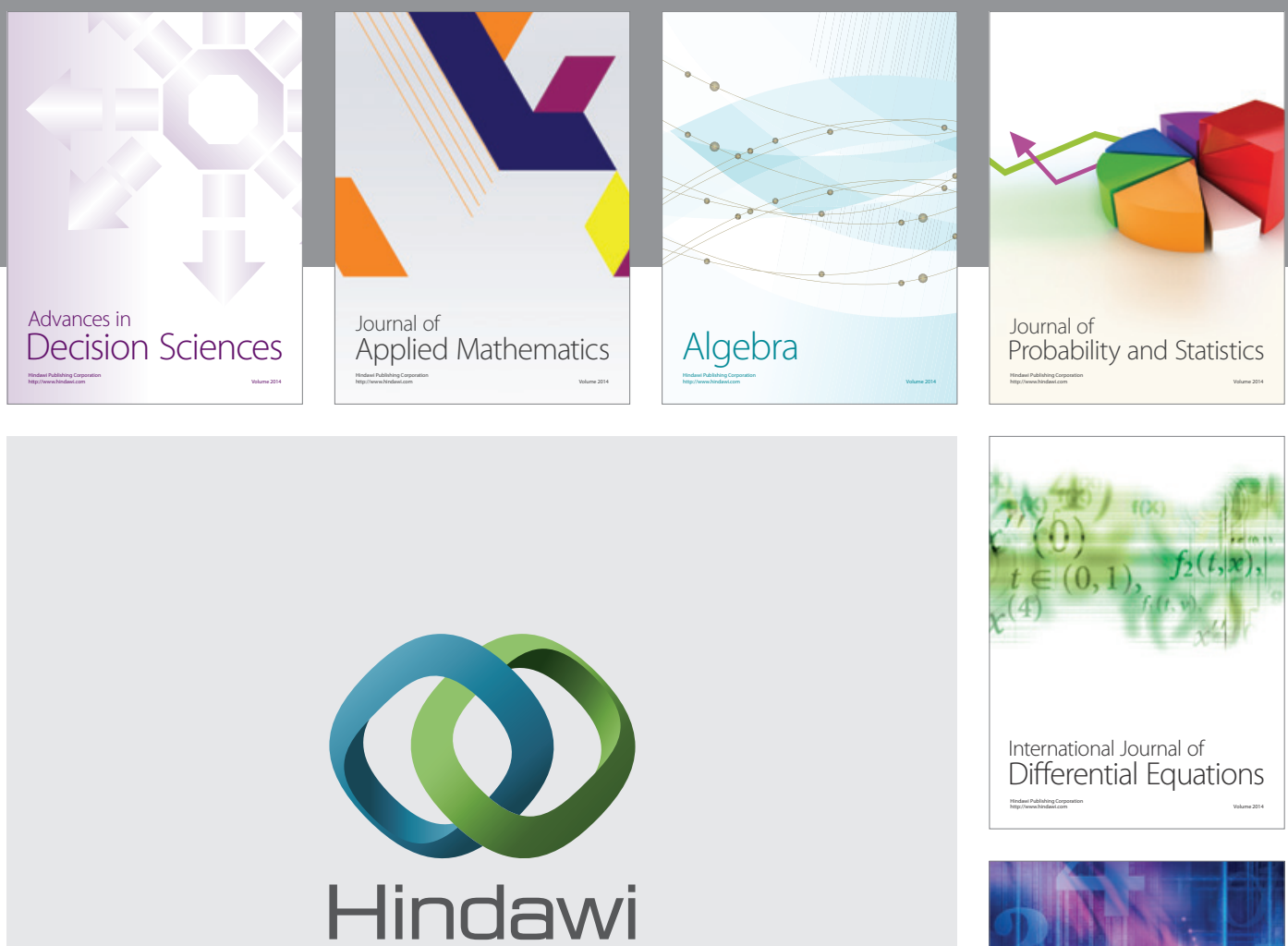

Submit your manuscripts at http://www.hindawi.com
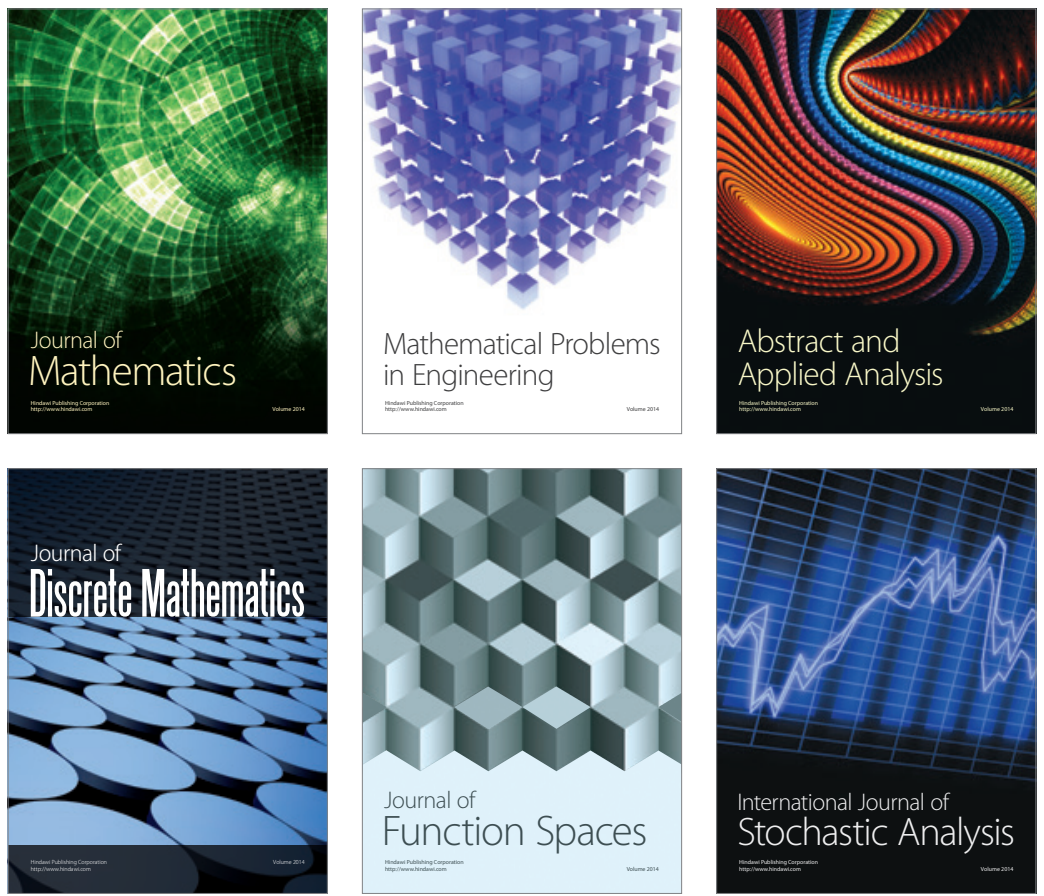

Journal of

Function Spaces

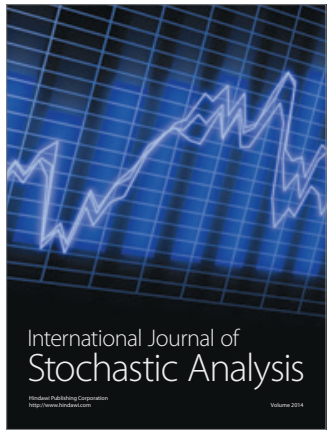

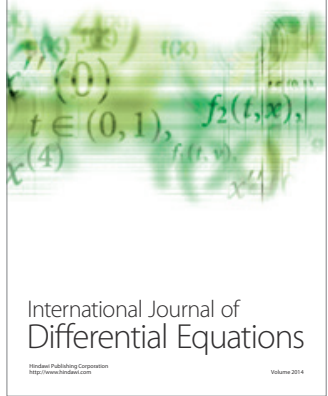
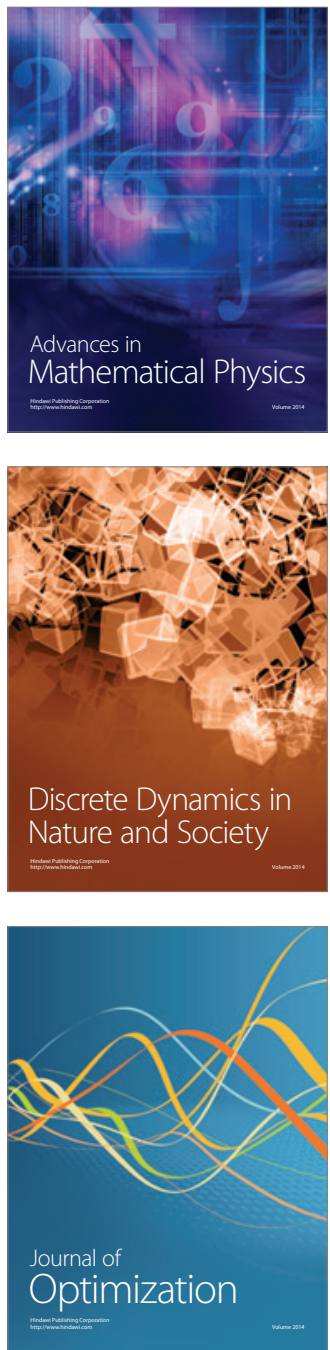